\title{
Global temperature as a regulator of climate predictability
}

\author{
A.A. Tsonis ${ }^{a}$,* J.B. Elsner ${ }^{b}$ \\ ${ }^{a}$ Department of Geosciences, University of Wisconsin-Milwaukee, Milwaukee, WI 53201-0413, USA \\ ${ }^{\mathrm{b}}$ Department of Meteorology, Florida State University, Tallahassee, FL 32306-3034, USA
}

Received 5 February 1996; accepted 2 December 1996

Communicated by $\mathrm{H}$. Flaschka

\begin{abstract}
The possibility of changes in the dynamical behavior of our climate system as the global temperature fluctuates is investigated by considering the large climate variation of the Pacific ocean-atmosphere system associated with Southern Oscillation Index (SOI) and modern methods of time series analysis. First the nonlinear structure of SOI is probed by estimating local Lyapunov exponents. We obtain the sum of all positive Lyapunov exponents (Kolmogorov entropy) as a function of time for the period between June 1882 and May 1987. We then demonstrate that this time series and global temperature records are coherent over the Nyquist frequency band from 0.0 to $0.25 \mathrm{cycles} /$ year thus establishing linear relationship between many of their oscillatory components. Since the inverse of the Kolmogorov entropy is a measure of predictability this result indicates that global temperature may affect predictability with higher temperatures corresponding to lower predictability. We discuss how this result may eventually lead to the development of strategies for improved long-term predictions.
\end{abstract}

\section{Introduction}

Fluctuations (natural or induced) of the global temperature can affect many of the outputs of the climate system such as precipitation, sea level, vegetation, ecosystems, etc. Because of the enormous significance of those outputs a major effort in determining the extent of such changes and their effects has been undertaken in the past fifteen years or so. Changes, however, in the outputs of the climate system reflect changes in the dynamics of the system. Thus, changes in the dynamical behavior of the climate system as the global temperature fluctuates can provide useful insights and reveal fundamental properties of the system.

\footnotetext{
* Corresponding author.
}

We chose to approach this problem by investigating how global temperature affects the predictability of the climate system. Toward this end we need to consider a strong signal of our climate system with demonstrated nonlincar structure. A good candidate is the SOI (Southern Oscillation Index) record. The complex nonlinear character of SOI has been investigated and documented over the last decade from several empirical studies [1-4]. One of the basic understandings from these and other studies is that SOI represents an output from a low-order chaotic dynamical system with a dimension as low as about 5 and a mean orbital period of about five years. Because of the established nonlinear character of SOI and its connection to global dynamics it presents an excellent candidate to empirically investigate the relation between predictability and global temperature. Note that often SOI 


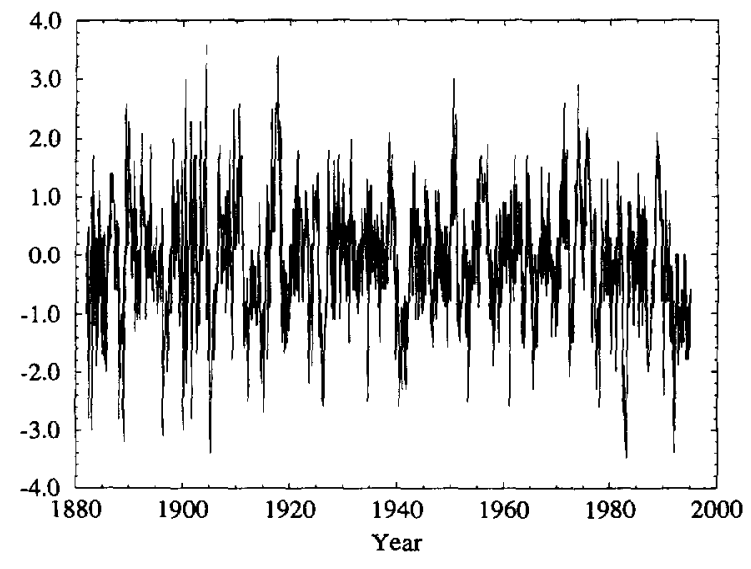

Fig. 1. Time series of the normalized monthly mean sea-level pressure difference between Tahiti and Darwin. The record reveals aperiodic fluctuations in the atmospheric Walker circulation over the tropical Pacific Ocean and is typically referred to as the Southern Oscillation Index (SOI). The record runs from January 1882 to January 1995 for a total of 1357 values.

is considered a characteristic of the $\mathrm{El} \mathrm{Niño/Southern}$ Oscillation (ENSO) state. However, a direct connection between SOI and ENSO dynamics [5-11] should be made with caution as SOI possibly includes other dynamics as well.

Dynamically speaking predictability is equal to the inverse of the Kolmogorov entropy $(K)$ which is equal to the sum of all the positive Lyapunov exponents. Here we assess predictability by probing the local structure of the attractor along the trajectory generated by the SOI record (Fig. 1). Our analysis is based on procedures that estimate local Lyapunov exponents along a trajectory on the attractor $[12,13]$. The local Lyapunov exponents govern the local (over a finite time) growth or decay of small perturbations to orbits of a dynamical system. They are closely connected to the intricacies of the structure of the corresponding attractor and are critical to assessing the predictability of the system.

\section{Data analysis and results}

We assumed a time delay $\tau=1$ and embedded the data in a six-dimensional space. This dimension is often referred to as the global embedding dimension, $d_{\mathrm{E}}$.
A delay of $\tau=1$ corresponds to lag 1 autocorrelation, $\rho(1)$. For SOI $\rho(1) \sim 0.5$. This is close to $1 / \mathrm{e}$ a value often used as the decorrelation time. Other choices of $\tau$ between 2 and 12 did not affect the results. Determination of the embedding dimension was guided by previous results $[1,8]$ and from nonlinear prediction $[14,15]$. Nonlinear prediction computes the correlation coefficient, $r(t)$, between the actual and predicted values as a function of the prediction time, $t$. As is suggested by Sugihara and May [15] an effective way to determine the proper embedding dimension is to compute $r(1)$ as a function of the embedding dimension and find at which embedding dimension $r(1)$ is maximized. In our case $r(1)$ was maximum at $d_{\mathrm{E}}=6$. This result is consistent with recent results that established nonlinear structure in the SOI $[3,4]$. Such an embedding results in $n=1352$ points with the first point, $y(1)$, having coordinates consisting of the first six values of the SOI record and the 1352nd point, $y$ (1352), having coordinates consisting of the last six values of the SOI record. We thus have a trajectory that is the sequence of points $y(1), y(2), \ldots, y(1352)$ which we presume to be governed by $y(i+1)=\mathbf{F}(y(i))$ where $\mathbf{F}$ is some mapping that takes $y(i)$ to $y(i+1)$. For a given point in our six-dimensional space the local Lyapunov exponents are estimated from the eigenvalues of the Jacobian of small deviations about the point (a small neighborhood). To evaluate the Jacobians we used linear neighborhood maps and a local embedding dimension [13], $d_{\mathrm{L}}$, of six. The number of forward iterates of the $6 \times 6$ Jacobian was determined by the decomposition length, $L$, which was taken to be equal to 72 . From all available points $y(i), i=1, n$ we were, therefore, able to obtain time series for six local Lyapunov exponents $\lambda_{j}(i), i=1, m, j=1,6$. Due to the choice of $d_{\mathrm{E}}=6$ and $L=72$ we estimated the local Lyapunov exponents for points $y(1)$ (which in the time domain would correspond to June 1882) to $y(1260)$ (which would correspond to May 1987). Thus, $m=1260$.

For SOI we find that there exist two positive exponents (indicating expansion along two directions) one zero exponent (corresponding to the slowly changing magnitude of the principal axis tangent to the flow) and three negative (indicating contraction along 
the remaining directions). The sum of the positive Lyapunov exponents is an estimate of the metric (Kolmogorov) entropy, $K$, and is related to the geometry of the underlying dynamics. The inverse of $K$ is a measure of the predictability of the system. For the two positive Lyapunov exponents we obtain that $\left\langle\lambda_{1}\right\rangle=(1 / m) \sum \lambda_{1}(i)=0.273$ (months $\left.^{-1}\right)$ and $\left\langle\lambda_{2}\right\rangle=0.130$ (months $^{-1}$ ). The fact that the two average values are comparable suggests that chaotic dynamics in SOI arise from the interference of two mechanism of instability of comparable importance. The sum $\lambda_{1}+\lambda_{2}$ ranges from a minimum of about 0.3 to a maximum of about 0.5 with $\left\langle\lambda_{1}+\lambda_{2}\right\rangle=0.403$ (months ${ }^{-1}$ ). These values put the limits of predictability $\left(\left[\lambda_{1}+\lambda_{2}\right]^{-1}\right)$ of the underlying dynamics in SOI (not necessarily of ENSO whose predictability limits are higher) between 2 and 3.3 months. Fig. 2 shows $\lambda_{1}+\lambda_{2}$ (or $K$ ) for points $y(1)$ to $y(1260$ ) (i.e. from June 1882 to May 1987). We will denote this function as $f_{K}(i), i=1,1260$.

The next step is to show that the above results are statistically significant and that most likely they are due to nonlinear structure in the SOI record. Toward this end we compared the SOI results with results from surrogate data. The surrogate data $[16,17]$ were produced by computing the Fourier transform of the raw data (SOI), then multiplying each complex amplitude by $\mathrm{e}^{i \phi}$ where $\phi$ is a random variable in the in-

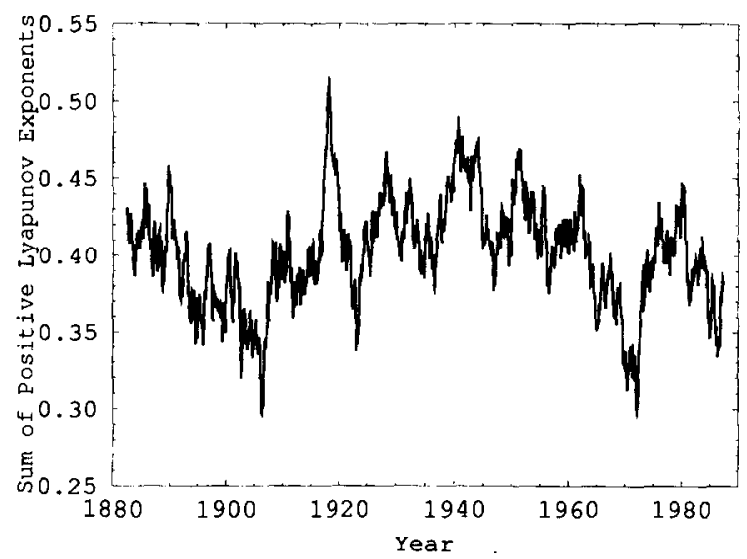

Fig. 2. The sum of the positive Lyapunov exponents (months ${ }^{-1}$ ) along the trajectory (or as a function of time) generated by the SOI record. The inverse of this sum is a measure of the predictability of the system (see text for details). terval $[0,2 \pi]$ and then take the inverse Fourier transform. Note that according to such a procedure the null hypothesis is that the data come from a linear gaussian process. The resulting random records have the same mean, variance and frequency distribution as the raw data. Unlike, however, surrogate data produced by shuffling the raw data, such surrogates preserve the autocorrelation structure of the raw data but not the dynamics. We generated one hundred surrogate SOI records (each of length 1357 values) and then we derived their $f_{K}(i), i=1,1260$ functions. For each of these functions we calculated $\langle K\rangle$ and then derived the probability distribution of $\langle K\rangle$ which is shown in Fig. 3(a). Note that in the case of the surrogates it is not rare that more than two positive Lyapunov exponents would exist. Here $K$ is the sum of all positive exponents not just the sum of the two largest exponents. The mean and standard deviation of this distribution are 0.434 and 0.0258 . From this figure we find that the probability that $\langle K\rangle$ will take on a value less or equal than 0.403 (i.e the SOI mean value) is 0.08 (eight surrogate records resulted in a value $\langle K\rangle \leq$ 0.403 ). This provides an estimate of $92 \%$ for the confidence level (one-sided test at which the null hypothesis that the SOI mean value is equal to the surrogates mean value is rejected. In order to make our case even stronger we performed additional tests. Specifically, we performed Fourier analysis on the $f_{K}(i), i=1$, 1260 functions of SOI and its surrogates. Statistically significant peaks form such functions will indicate mean orbital periods. For SOI the analysis indicates the presence of rather weak peaks at several frequencies (bold line in Fig. 3(b)). Next we considered the spectral density functions of all the surrogates and obtained at each frequency a distribution for the spectral density. The thin lines in Fig. 3(b) show the 10 and 90 percentiles of these distributions. From this figure it appears that the most significant of those peaks (significance level $>90 \%$ ) is centered at a frequency of about 0.015 cycles/month. This indicates a mean orbital period in SOI of 5-6 years which is what one would expect. The recovery of this result provides additional confidence that the results in Fig. 2 are not consistent with linear gaussian noise and that most likely are due to nonlinear structure and dynamics in the SOI record. 

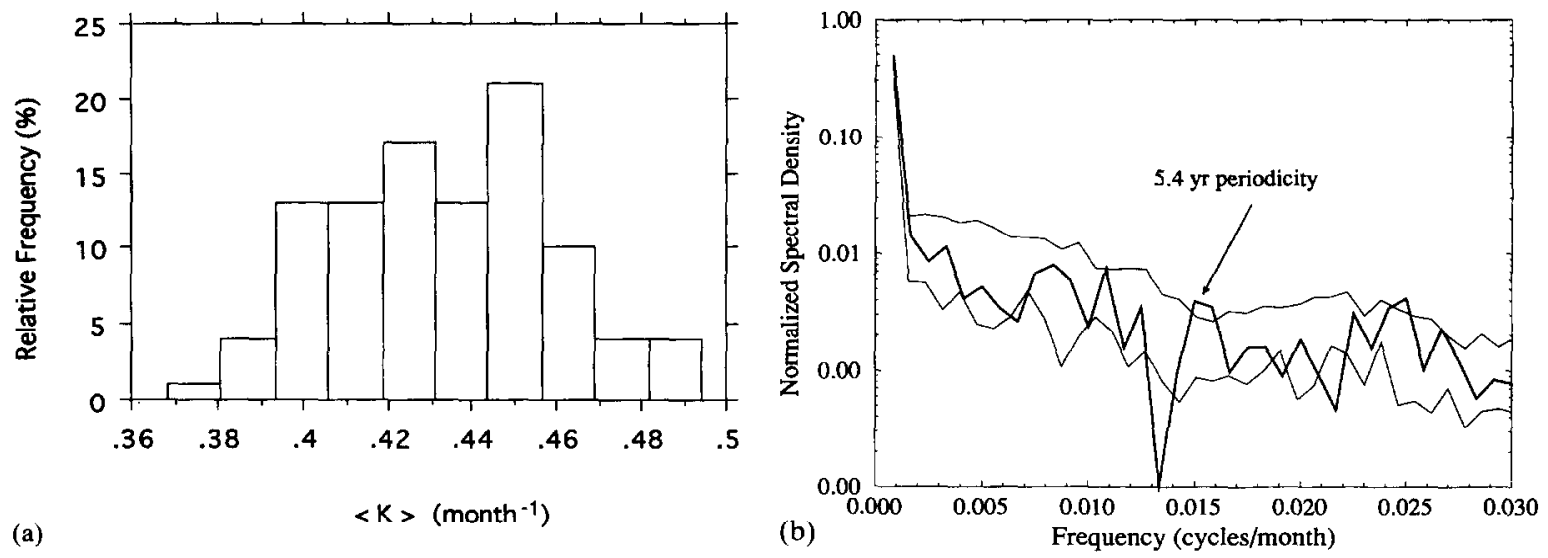

Fig. 3. Surrogate data analysis: (a) For each surrogate record we calculated $\langle K\rangle$ and then derived the probability distribution of $\langle K\rangle$ which is shown here. The brackets indicate averages. From this figure we find that the probability that $\langle K\rangle$ will take on a value less or equal than 0.403 (i.e. the SOI mean value) is approximately 0.08 (eight surrogate records resulted in a value $\langle K\rangle \leq 0.403$ ). This provides an estimate of $92 \%$ for the confidence level at which the null hypothesis that the SOI mean value is equal to the surrogates mean value is rejected. (b) The bold line is the spectral density function of $f_{\mathrm{K}}$ for SOI. By considering the spectral density functions of all the surrogates we obtain at each frequency a distribution for the spectral density, The thin lines in Fig. 3(b) show the 10 and 90 percentiles of these distributions. From this figure it appears that the most significant peak (confidence level of $\sim 90 \%$ ) is centered at a frequency of about 0.015 cycle/month. This indicates a mean orbital period in SOI of five to six years which is what one would expect.

Employing other procedures for surrogate data generation that extended the null hypothesis to consider the possibility of monotonic nonlinear transformation of a linear gaussian noise $[16,17]$ did not alter the above conclusion. Note that nonlinear structure in the SOI record has also been suggested from nonlinear prediction on the SOI and its surrogates [3,4].

A careful examination of Fig. 2 reveals striking similarities with global temperature records [18-21] as it exhibits an overall positive trend with the following features: a decrease up to about 1905, a steady increase up to about 1940, a subsequent decrease up to about 1970 and a rise afterwards. Such features can be identified in almost all global temperature records as for example in the global marine air temperature record [21] (Fig. 4). These two records correlate highly but this could be due to the presence of the overall slight positive trends. If, however, it is found that the detrended series are coherent over a band of frequencies, then it is more likely that the two time series are related. Coherence [22] is a measure of the linear correlation between two time series over a given frequency band when the phase difference is set to zero. Statistically

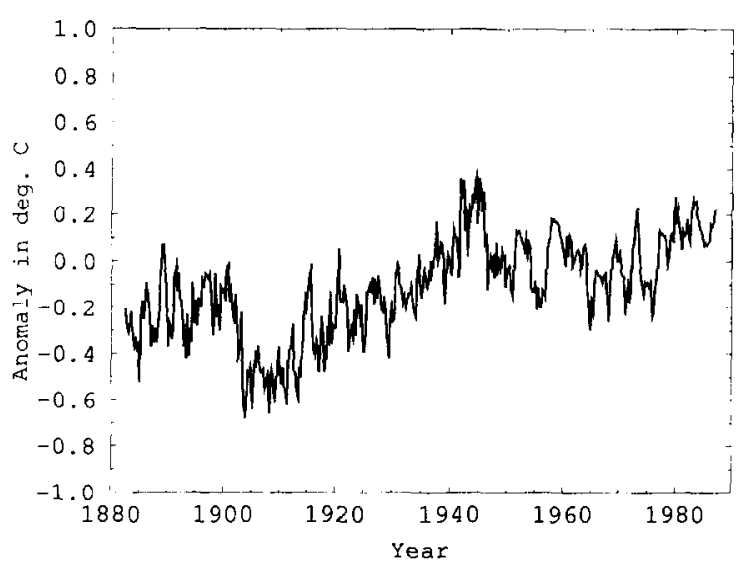

Fig. 4. The global marine air temperature record. Comparison with Fig. 2 reveals striking similarities between the two signals. Both series have an overall slight positive trend and segments of similar tendencies.

significant coherence over a frequency band indicates linear relationship between the corresponding oscillatory components. In addition, phase estimates can provide temporal (lead/lag) relationships between the two variables. Thus, coherence estimates and phase 


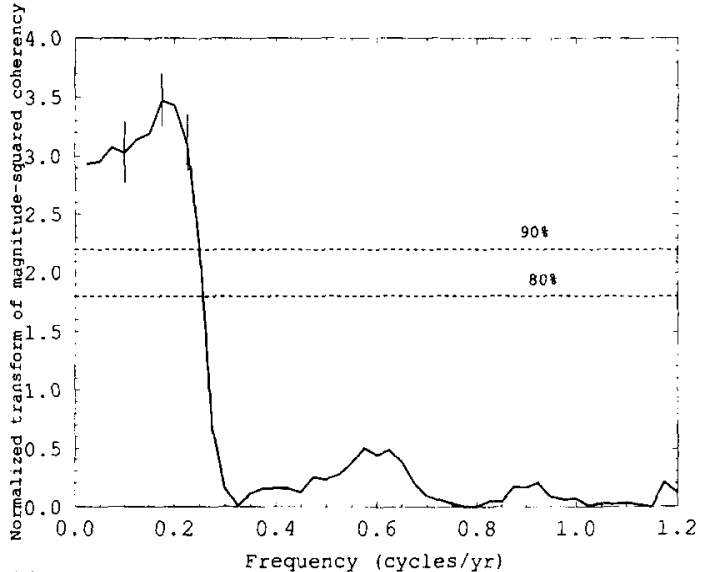

(a)

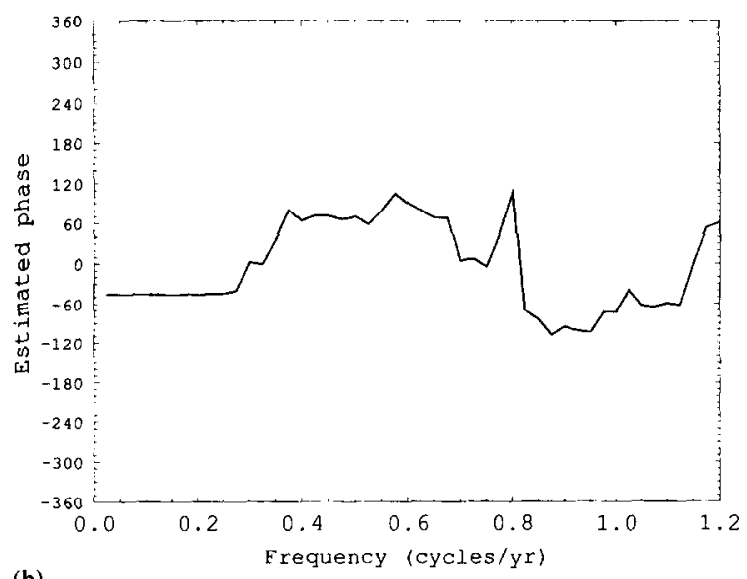

(b)

Fig. 5. (a) Magnitude-squared coherence between the signals in Fig. 2 and 4 transformed by $\tanh ^{-1}$. On this scale the values are gaussian with unit standard deviation. The $80 \%$ and $90 \%$ confidence levels of this distribution are shown by the parallel broken lines. The bars show one standard deviation of the transformed coherence values as determined by jack-knifing over windows [24] for selected frequencies. (b) Phase of coherence between the two signals. 'The absence of any significant trends indicate no lead/lag relationships. The graphs are not extended to higher frequencies because the coherence remains almost zero every where.

relationships can provide useful insights about physical relationships.

Fig. 5(a) shows the multiple-window [23,24] magnitude-squared coherence of the detrended time series transformed by $\tanh ^{-1}$. As a compromise between statistical reliability and resolution we adapted a bandwidth of 0.16 cycles/year which results in 16 Slepian sequences $(0.16 \times T)$ where $T=105$ is the length of the series in years). On such a scale coherence estimates are roughly gaussian with unit standard deviation [23]. As a consequence, the statistical significance of the coherence between the two series can be made directly. The $80 \%$ and $90 \%$ confidence levels of this distribution are shown as the two horizontal lines. Remarkably the two signals have a coherence much above the $90 \%$ confidence level at frequencies < 0.25 cycles/year (i.e. for time scales greater than about four years). Thus, the residuals of the two series are coherent with high confidence over the above low-frequency band. At higher frequencies apparently other unknown factors inhibit high coherence. Phase estimates (Fig. 5(b)) show no positive or negative trend suggesting no lead/lag relationships between the two signals. We can thus conclude that warmer temperatures correspond to higher $K$ values or to lower predictability. Similar conclusions are drawn when other global temperature records $[18,20]$ are considered. Therefore, there is significant evidence that global temperature and Kolmogorov entropy, $K$, are linearly related at all frequencies lower than 0.25 cycles/year. Thus, as the temperature of the planet increases predictability $(1 / K)$ of the climate system decreases. Note that recent studies have raised the question as to what happens to entities like ENSO under climate change scenarios [25], but they have concentrated on parameters such as the amplitude of its cycle. Our work deals with the more fundamental question of whether or not predictability regulators exist in the climate system.

\section{Conclusions}

Dynamically speaking we may view global temperature as a controlling factor (input) whose changes modify the dynamics and thus the character of the system, a view consistent with the theory of connected dynamical subsystems [26]. Physically, it is possible that poor predictive skill is due to increased variability of the system. As has been suggested by model simulations $[27,28]$ higher temperature may result in intensification of the extremes of the hydrological cycle, in enhanced convective activity and other changes that 
can alter the spatial variability of the system and thus its predictability (yet another pitfall of global warming). On the brighter side our result offers the suggestion that better long-term predictions (on scales of several years to decades) may be anticipated if those predictions are made during the cooler phases of our climate system. As such, and especially if other factors that relate to predictability can be found, extension of the type of analysis presented here may lead to strategies for better long-term predictions. Research in this area is in progress and results will be reported later elsewhere.

\section{Acknowledgements}

This work has been supported by NSF grants ATM9310959 to A.A. Tsonis and ATM-9310715 to J.B. Elsner.

\section{References}

[1] A. Hense, Beitr. Phys. Atmosph. 60 (1987) 34-47.

[2] S.T. Bauer and M.B. Brown, Geophys. Res. Lett. 19 (1992) 2055-2058.

[3] A.A. Tsonis and J.B. Elsner, Nature 35 (1992) 217-220.

[4] J.B. Elsner and A.A. Tsonis, Geophys. Res. Lett. 20 (1993) 213-216.

[5] G.K. Vallis, Science 232 (1986) 243-245.

[6] P. Chang, B. Wang, T. Li and L. Ji, Geophys. Res. Lett. 21 (1994) 2817-2821.

[7] F.-F. Jin, J.D. Neelin and M. Ghill, Science 264 (1994) $70-72$.
[8] E. Tziperman, L. Stone, M.A. Cane and H. Jarosh, Science 264 (1994) 72-74.

[9] M.A. Cane and S.E. Zebiak, Science 228 (1985) 10851089.

[10] S.E. Zebiak and M.A. Cane, Mon. Weather Rev. 115 (1987) 2262-2281.

[11] S.G.H. Philander, El Niño, La Niña and the Southern Oscillation (Academic Press, San Diego, 1990).

[12] H.D.I. Abarbanel, R. Brown and M.B. Kennel, Int. J. Mod. Phys. 5 (1991) 1347-1375.

[13] R. Brown, P. Bryant and H.D.I. Abarbanel, Phys. Rev. A 43 (1991) 2787-2806.

[14] J.D. Farmer and I.J. Sidorowich, Phys. Rev. Lett. 59 (1987) 845-848.

[15] G. Sugihara and R.M. May, Nature 344 (1990) 734 741.

[16] J. Theiler, S. Eubank, A. Longtin, B. Galdrikian and J.D. Farmer, Physica D 58 (1992) 77-94.

[17] L.A. Smith, Physica D 58 (1992) 50-76.

[18] J.E. Hansen and S. Lebedeff, J. Geophys. Res. 92 (1987) $13345-13372$.

[19] P.D. Jones, T.M.L. Wigley and P.B. Wright, Nature 322 (1986) $430-434$

[20] C.K. Folland, T.R. Karl and K.Ya. Vinnicov, Climate Change. The IPCC Scientific Assessment, eds. J.T. Houghton, G.J. Jenkins and J.J. Ephramus (Cambridge University Press, Cambridge, 1990).

[21] N.E. Newell et al., Geophys. Res. Lett. 16 (1989) 311-314.

[22] M.B. Priestley, Spectral Analysis and Time Series (Academic Press, New York, 1981).

[23] D.J. Thomson, Proc. IEEE 70 (1982) 1055-1096.

[24] S. Kuo, C. Lindberg and D.J. Thomson, Nature 343 (1990) $709-714$.

[25] D. Gu and S.G.H. Philander, J. Climate 8 (1995) 864-876.

[26] A.A. Tsonis, Complexity 1 (1996) 23-33.

[27] J. Hansen et al., Proc. 2nd North American Conf. on Preparing for Climate Change (Climate Institute, Washington, 1988).

[28] K.A. Emanuel, Nature 326 (1987) 483-485. 